\title{
Villoglandular Adenocarcinoma of Urethra with Negative CK7 Marker: Rare Disease in Atypical Location
}

ISSN: 2578-0395

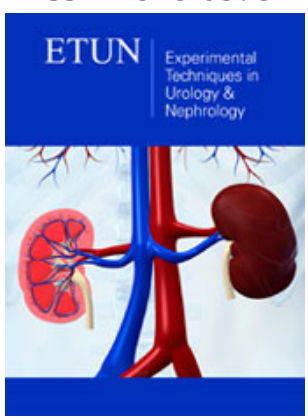

*Corresponding author: Matheus WE, From the Departments of Surgery and Division of Urology, School of Medical Sciences, Brazil

Submission: 啙 August 05, 2019

Published: 海September 19, 2019

Volume 3 - Issue 1

How to cite this article: Matheus $\mathrm{W}$ Ferruccio A, Matheus M, Almeida B D, Ferreira U. Villoglandular Adenocarcinoma of Urethra with Negative CK7 Marker: Rare Disease in Atypical Location. Exp Tech Urol Nephrol. 3(1). ETUN.000551.2019. DOI: 10.31031/ETUN.2019.03.000551

Copyright@ Matheus WE, This article is distributed under the terms of the Creative Commons Attribution 4.0 International License, which permits unrestricted use and redistribution provided that the original author and source are credited.
Matheus WE*, Ferruccio AA, Matheus MB, Almeida Braga D and Ferreira U

Departments of Surgery and Division of Urology, Brazil

\section{Abstract}

Urethral cancer is a rare disease that affects predominantly males and the black race. The case report refers to a female patient of 75 years who had a history of dysuria and urethral pain. After an investigation with urethrocytoscopy and biopsy, a histopathological diagnosis of villograndular adenocarcinoma was identified. The immunohistochemical study identified the presence of beta-catenin and the absence of CK7 expression. In a literary review, few cases of this particularly histological type where published about the urethra and none of them exhibited the tumor marker CK7 negative.

Keywords: Adenocarcinoma; Urethra; Villograndular; CK7

\section{Introduction}

Urethral cancer is a rare disease representing less than $1 \%$ of genitourinary tumors. It affects with a higher frequency male and the black race [1]. Studies show that its estimated prevalence in North America is 4.3 cases per million men and 1.5 cases per million women. The World Health Organization (WHO) histologically classifies the urethral tumors as urothelial carcinoma, squamous cell carcinoma and adenocarcinoma [2], this last one with an incidence lower than $5 \%$ of the cases [3]. The urethral neoplasm is considered challenging to diagnose, due to the nonspecific symptoms it may present such as dysuria, hematuria, polaciuria and urinary retention. These symptoms may be confused with urinary infection and lead to a diagnosis in advanced stages of the disease $[1,3]$. The treatment depends primarily on the location and the stage of the tumor, being its elementary principle the complete tumor resection. In this report, we presented the case of a woman diagnosed with a rare type of neoplasm: villograndular adenocarcinoma, with the immunohistochemical study presenting beta-catenin positive and CK7 marker negative. After careful evaluation, the treatment designated for the tumor being discussed was urethrotomy in association with cystostomy, diverging from the standard recommendations due to the staging of the disease.

\section{Case Report}

A 75-year-old female patient had dysuria, macroscopic hematuria and urethral pain for 1 year. She denied supra-pubic pain, effort to urinate and other urinary complain. From her past, denied smoking and alcoholism, but had a history of resection of squamous cell carcinoma of the vulva, severe heart failure, systemic arterial hypertension, idiopathic pulmonary fibrosis and multiple thyroid nodules. Regarding the use of medications, she made continuous use of losartan, furosemide, atenolol, spironolactone, acetylsalicylic acid, folic acid, methotrexate and prednisone. At the physical exame, presented an exophytic and friable vegetative lesion that projected through the urethral meatus. A biopsy of the urethral lesion through transurethral resection was performed, as well as a tumor staging with computed tomography of the chess and abdomen, cystoscopy and magnetic resonance imaging of the pelvis. The fragments removed demonstrated in the histopathological evaluation villoglandular adenocarcinoma of urethra with superficial focal invasion of the connective tissue (pT1). A pelvic Magnetic Resonance showed a concentric and irregular urethral thickening of tumor aspect, involving the entire urethra with approximately $4.0 \mathrm{~cm}$ in length and a maximum thickness of $1.2 \mathrm{~cm}$. The 
lesion was flat, immediately below the internal ostium, reached the perineal area, of heterogeneous aspect, mainly solid, with diffusion restriction and predominant in the right anterolateral region. Cystic and necrotic areas were present in close contact with the anterior wall in the distal third of the vagina and bladder floor.

There were also no signs of invasion of adjacent organs and fat in the retro pubic space. The inguinal lymph nodes showed bilateral heterogeneous characteristics, measuring up to $1.0 \mathrm{x}$ $0.7 \mathrm{~cm}$, being described as undetermined Initially, classic treatment of radical cystectomy and complete urethrectomy (anterior pelvic exenteration) was proposed. However, due to the patient's poor clinical conditions, she only went through a complete urethrectomy associated with cystostomy (Figure 1). The surgical approach was abdominal and perineal through spinal anesthesia, lasting approximatey 2 hours and estimated bleeding of $300 \mathrm{ml}$. During the surgery, the patient presented significant bradycardia, requiring atropine and vasoactive drugs, with complete reversal of the hemodynamic scenery. The anatomopathological analysis (Figure 2) concluded the diagnosis of focally mucinous urethral adenocarcinoma (with villoglandular feature in previous biopsy) and invasion of the periurethral muscle layer, pT2. The surgical margins were free of neoplasia and the immunohistochemical study revealed the presence of positive Beta-catenin and absence of CK7 marker expression. The patient presented satisfactory clinical evolution, during the postoperative period. There were no complications during the hospitalization and the discharge occurred on the 5th postoperative day. The patient is in the 8th postoperative month, with good surgical healing, good urinary volume through cystostomy and no apparent pelvic recurrence.

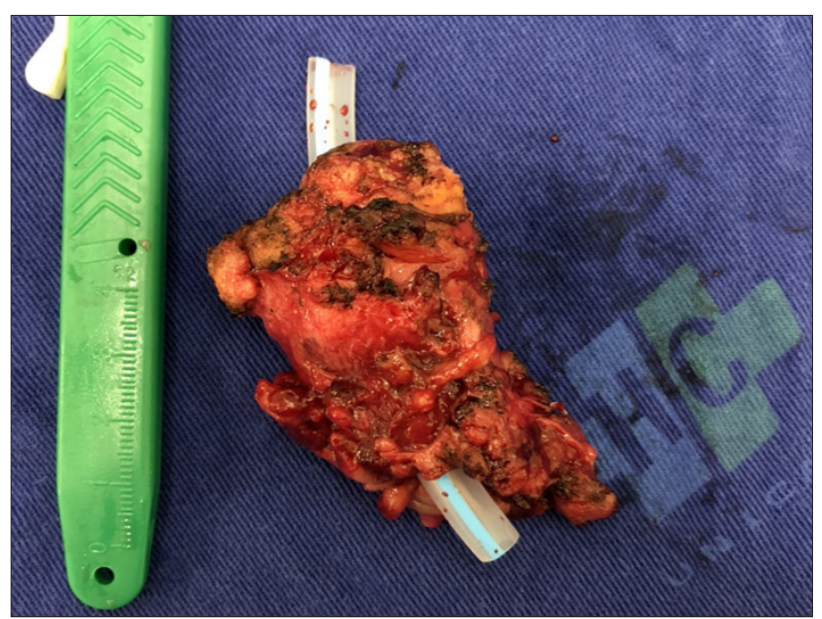

Figure 1: Fragment of urethra containing the tumor.

\section{Discussion}

The urethral carcinoma is a rare and essentially aggressive disease that represents less than $1 \%$ of genitourinary tumors. In females, the estimated prevalence of these tumors is only at $0.02 \%$ of the studied cases. Urothelial carcinoma and squamous cell carcinoma being the most common types in this group of patients $[1,3,4]$. Due to the low incidence and aggressiveness of the tumor, the knowledge gathered about urethral adenocarcinomas is mainly through case reports $[1,3,5]$. The immunohistochemical analysis was performed in few described cases, which makes the correlations between this tumor and its markers difficult. In some reports, the association between Beta-catenin in the cell nucleus and intestinal adenocarcinomas were described, while the tumor marker CK7 is closely related to the urothelial component of urothelial carcinomas with villoglandular component [6]. Diagnosing this disease is a challenge due to the similarity between symptom presented by it and to other illnesses that are more common, such as urinary infection $[1,3]$. By the time the tumor is discovered, the vast majorities are in an advanced stage and allow few therapeutic proposals. The base of the treatment depends on the oncological staging of the tumor and consists mainly in a complete resection surgery, which may be associated or not with radiotherapy or chemotherapy $[3,4]$. A clinical case of a white female was described in this study, this way, the demographic characteristics were not consistent with the epidemiological predisposition of this disease $[1,7,8]$. Although the information does not match literature, the unspecific clinical conditions of the patient, throughout the months, was a match with the history of other cases. The most significant moment for the diagnosis was the appearance of exophytic lesion in the urethra.

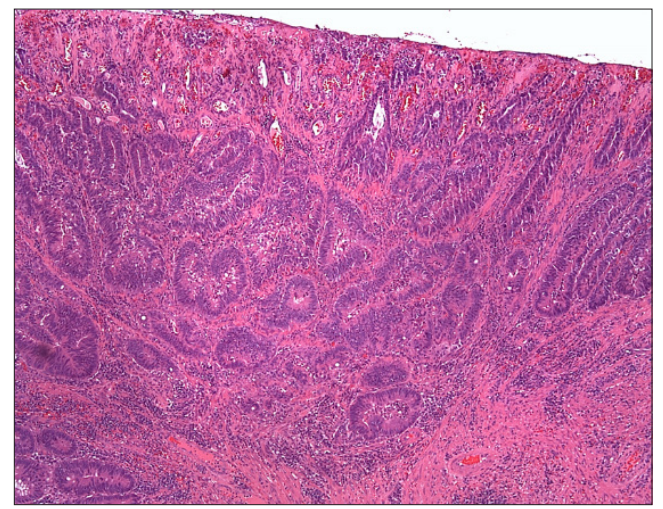

Figure 2: Atypical villoglandular presence in a urethral fragment.

The anatomopathological diagnosis reveled atypical presentation of urethral cancer. The adenocarcinoma with the presence of villoglandular characteristics, even more rare in this infrequent tumor subtype $[2,6,7]$. Immunohistochemical analysis was also surprising due to the positive presentation of Beta-catenin and negative CK7 marker, not compatible with literature data [611]. In this atypical case, the possibility of relating the appearance of this tumor to the immunosuppression that the patient suffered for the treatment of idiopathic pulmonary fibrosis was considered. Staging was classified as pT2 by TNM, with no lymph nodes or metastasis detected neither through image exams nor on the analysis of the biopsied material. The recommended treatment for this staging, according to the National Comprehensive Cancer Network guidelines, is urethrectomy associated with cystectomy. However, during the cautious surgical planning performed by the medical team, the therapeutic proposal was urethrectomy associated with cystostomy, preserving the bladder due to the 
patient's poor clinical condition. After the surgical procedure, the patient had good clinical parameters and had a good recovery on the postoperative period, consistent with literature data.

\section{Conclusion}

Urethra adenocarcinoma is a rare condition, especially in this patient's case, due to the villoglandular presence, with positive beta-catenin and no expression of the CK7 marker on the immunohistochemical approach. The conduct when dealing with this diagnosis is radical cystectomy and urethrectomy surgery, well established by worldwide protocols, however there are clinical, histopathological and immunohistochemical characteristics that challenge professionals to make decisions regarding the best treatment for these patients. Precisely, given their low incidence, case reports are of extreme relevance to improve our knowledge of this rare pathology.

\section{References}

1. Dayyani F, Hoffman K, Eifel P, Guo C, Vikram R, et al. (2014) Management of advanced primary urethral carcinomas. BJU Int 114(1): 25-31.

2. Zhang M, Adeniran AJ, Vikram R, Tamboli P, Pettaway C, et al. (2018) Carcinoma of the urethra. Human Pathology 72: 35-44.

3. Basiri A, Narouie B, Moghadasi MH, Ghasemi Rad M, Valipour R (2015) Primary adenocarcinoma of the urethra: a case report and review of the literature. Journal of Endourology Case Reports 1(1): 75-77.
4. Grigsby, Perry W (1998) Carcinoma of the urethra in women. International Journal of Radiation Oncology Biology Physics 41(3): 535-541.

5. Derksen JW, Visser O, De la Rivere GB (2013) Primary Urethral carcinoma in females: An epidemiologic study on demographical factors, histological types, tumour stage and survival. Word J Urol 31(1): 147153.

6. Mitra S, Chatterjee D, Das A, Gupta K, Radotra BD, et al. (2018) Urothelial tumors with villous morphology: Histomorphology and role of immunohistochemistry in diagnosis. APMIS 126(3): 191-200.

7. Swartz, Mia A, Porter MP, Lin DW, Weiss NS, et al. (2006) Incidence of primary urethral carcinoma in the United States. Urology 68(6): 11641168.

8. Visser O, Adolfsson J, Rossi S, Verne J, Gatta G, et al. (2012) Incidence and survival of rare urogenital cancers in Europe. European Journal of Cancer 48(4): 456- 464

9. Neto C, José de, Leão, Sydney C., Fakhouri, Ricardo, Gurgel, Roberto, et al. (2016) Adenocarcinoma of the female urethra: a case report. Jornal Brasileiro de Patologia e Medicina Laboratorial 52(4): 266-269.

10. (2018) National Comprehensive Cancer Network. NCCN Clinical Practice Guidelines in Oncology: Bladder Cancer.

11. Libby B, Chao D, Schneider BF (2010) Non-surgical treatment of primary female urethral cancer. Rare tumors 2(3): e55. 